\title{
INITIAL TESTS OF MiniBETA SPECTROMETER PERFORMANCE*
}

\author{
M. Perkowski ${ }^{\mathrm{a}}$ b, K. Bodek ${ }^{\mathrm{b}}$, L. De Keukeleere ${ }^{\mathrm{a}}$, L. Hayen $^{\mathrm{a}}$

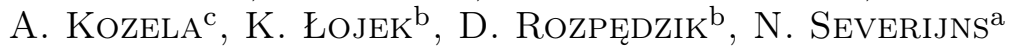 \\ ${ }^{a}$ KU Leuven, Department of Physics and Astronomy \\ Instituut voor Kern- en Stralingsfysica, 3001 Leuven, Belgium \\ ${ }^{\mathrm{b}} \mathrm{M}$. Smoluchowski Institute of Physics, Jagiellonian University \\ 30-348 Kraków, Poland \\ ${ }^{c} \mathrm{H}$. Niewodniczański Institute of Nuclear Physics, Polish Academy of Sciences \\ 31-342 Kraków, Poland
}

(Received January 12, 2018)

MiniBETA is a new spectrometer incorporating a low-pressure multiwire drift chamber (MWDC). It is designed for beta spectrum shape measurements and to improve knowledge on electron backscattering in the about $100 \mathrm{keV}$ to a few $\mathrm{MeV}$ region. After the commissioning phase, which we report on here, the spectrometer will first be used for the latter purpose. This will help reducing the systematic uncertainty in beta spectrum shape measurements. The performance of the trajectory recognition algorithm was investigated using cosmic muons as the source of the primary ionization. For the initial test, the chamber was filled with a mixture of helium-isobutane (50/50) at 600 mbar. A single cell efficiency $\eta$ higher than 0.98 within almost the entire cell was achieved and the single cell spatial resolution in the drift plane reached $\sigma=0.4 \mathrm{~mm}$ for most of the cells in the chamber.

DOI:10.5506/APhysPolB.49.261

\section{Introduction}

Precision measurements of the $\beta$-spectrum shape are an important method in the search for physics beyond the Standard Model [1]. They look for the existence of the Fierz interference term, which expands the SM by currents not included in the V-A theory. A non-zero value of this term would manifest itself as a slope in the ratio of the measured shape and the

* Presented at the XXXV Mazurian Lakes Conference on Physics, Piaski, Poland, September 3-9, 2017. 
one simulated according to the SM. Achieving a precision at the level of $10^{-3}$ on the Fierz term would allow reaching a sensitivity comparable to that of the Large Hadron Collider [2].

A persistent difficulty of shape measurements is the backscattering of electrons from detectors, which distorts the measured spectra. This effect is difficult to control as it depends on the energy and on the detector material [3]. The MiniBETA spectrometer allows to directly observe the backscattered electrons and provide empirical data for validation of the Monte Carlo calculation (e.g. Geant4 simulations) of corrections to be applied. It consists of a compact, modular, reconfigurable drift chamber in hexagonal geometry. The spectrometer readout is triggered by plastic scintillators and controlled by a dedicated data acquisition (DAQ) system [4, 5]. The $\beta$ source is mounted inside the chamber in a motorized, computer-controlled positioning system and can be exchanged via a sluice without breaking the gas conditions inside. The 3D electron track is deduced from the drift times ( $X, Y$ coordinates in the plane perpendicular to wires) and from the asymmetry of charge collected at the wire ends ( $Z$ coordinates, "charge division" technique) in the cells crossed by the electron.

The chamber consists of 80 hexagonal cells arranged in 10 rows. The hexagonal cell geometry has been chosen to reduce the amount of field wires needed to form a cell, hence reducing the probability of a $\beta$ particle hitting wires. The chamber is operating with a light noble gas (helium) mixed with a quencher (isobutane) at low pressure to minimize the effect of electron scattering in the gas. In the first step of the spectrometer characterization, a measurement of cosmic muon trajectories was performed in order to validate the particle tracking technique in the chamber and to calculate the individual cell efficiencies and their spatial resolution. The mixture ratio was set to $50 / 50$ at the pressure of 600 mbar.

\section{Drift time to drift distance relation}

The DAQ system records the drift time of the fastest ionization electron cluster generated along the charged particle path in each cell. One can calculate the distance $r$ between the anode (cell center) and the position where the particle trajectory is the closest, based on the drift time value and the electric field map (assuming radial symmetry). In order to obtain the best results, one has to verify this relation experimentally.

Calibration measurements using cosmic muons as a source of primary ionization in the gas mixture have a few advantages. Cosmic muons are highly energetic particles and their scattering by gas particles can be neglected, hence they maintain straight trajectories inside the drift chamber. They also provide an approximately homogeneous flux, which is beneficial for the calibration. 


\subsection{Initial calibration}

Under the assumption of a homogeneous flux of ionizing particles through the cell $\left(\mathrm{d} N / \mathrm{d} r=\right.$ const $=N_{0} / R_{\max }$, where $N_{0}$ is the total number of detected particles and $R_{\max }$ is the radius of an incircle of the cell), the following transformation can be used:

$$
v(t)=\frac{\mathrm{d} r}{\mathrm{~d} t}=\frac{\mathrm{d} r}{\mathrm{~d} N} \frac{\mathrm{d} N}{\mathrm{~d} t}=\frac{R_{\max }}{N_{0}} \frac{\mathrm{d} N}{\mathrm{~d} t},
$$

where $v(t)$ is the drift velocity, $r$ is the drift distance, $t$ is the drift time and $N$ is the number of muons.

Integrating Eq. (1) leads to the initial $r(t)$ calibration

$$
r(t)=\int_{t_{0}}^{t} v\left(t^{\prime}\right) \mathrm{d} t^{\prime}=\frac{R_{\max }}{N_{0}} \int_{t_{0}}^{t} \frac{\mathrm{d} N}{\mathrm{~d} t^{\prime}} \mathrm{d} t^{\prime} .
$$

Due to the finite spatial resolution, there are no clear edges in the spectrum, which could be related to the trajectories passing closest to the signal wire $\left(t_{0}\right)$ and closest to cell edges $\left(t_{\max }\right)$. They can be derived from the following empirical formula describing the drift time distribution in a tube-like cell [6]:

$$
\frac{\mathrm{d} n}{\mathrm{~d} t}=P_{0}+\frac{P_{1}\left(1+P_{2} \exp \left(\frac{P_{4}-x}{P_{3}}\right)\right)}{\left(1+\exp \left(\frac{P_{4}-x}{P_{6}}\right)\right)\left(1+\exp \left(\frac{x-P_{5}}{P_{7}}\right)\right)},
$$

where $P_{i}$ for $i=0, \ldots, 7$ are fit parameters: $P_{0}$ is the noise offset, $P_{1}$ is the scale factor, $P_{4}$ and $P_{5}$ correspond to $t_{0}$ and $t_{\max }, P_{2}$ and $P_{3}$ depend on the shape of the distribution, while $P_{6}$ and $P_{7}$ describe its leading and trailing edge slopes. Figure 1 (left) shows a typical ${ }^{1}$ drift time distribution for the measurement using cosmic muons with the corresponding shape fitted. The fit quality and the obtained parameters are not discussed in this paper because the values of $P_{4}$ and $P_{5}$ are only used for a guess of the initial calibration function.

Integrating the spectrum according to Eq. (2), in the range between $t_{0}$ and $t_{\max }$, leads to an initial $r(t)$ calibration. A sixth order polynomial was fitted to parametrize the calibration function. For $t<t_{0}$ and $t>t_{\max }$, the drift distance was set constant: $r\left(t<t_{0}\right)=0$ and $r\left(t>t_{\max }\right)=R_{\max }$. Figure 1 (right) shows the initial calibration function.

\footnotetext{
${ }^{1}$ In this paper, if an example of a cell performance (e.g. drift time spectrum, $r(t)$ calibration function, efficiency etc.) is presented, it refers to the cell defined by plane 9 wire 5 (the mapping can be seen in Fig. 5 (right)).
} 

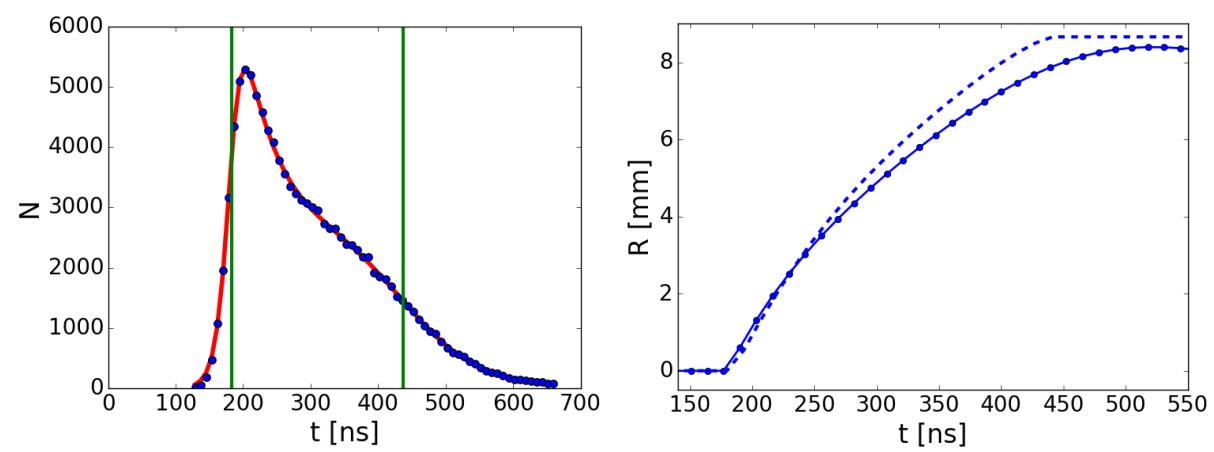

Fig. 1. Left panel: A typical drift time spectrum of a single cell with the distribution shape fitted (Eq. (3)). Vertical lines indicate the values of the parameters $P_{4}$ $\left(t_{0}\right)$ and $P_{5}\left(t_{\max }\right)$ obtained from the fit. Right panel: An example of the $r(t)$ calibration. The dashed line represents the initial calibration obtained by integrating the drift time spectrum. The solid line is the final result of the iterative calibration procedure (see Sec. 2.3).

\subsection{D tracking}

The DAQ system records digitized signals originating from the drift chamber within $1.5 \mu \mathrm{s}$ after receiving the trigger signal. All signals are marked with the individual cell number and the time stamp so that the separate drift time distributions can be built for individual cells and a track can be identified through signals having the same time stamp. The calibration procedure begins with a prefit of a straight line applied to the centers of the hit cells. The prefit provides the initial parameters for the main fit. This approach not only speeds up the optimization process but is also crucial for the stability of the main fit.

The main fit finds a straight line $A x+y+C=0$, closest to the circles originating in the cells centers with the radii calculated according to the $r(t)$ calibration functions, using the method of least squares. The fit residuum $\Delta_{i}$ ( $i$ denotes individual indices of the cells creating the event $)$ is defined as a difference between the calculated radius $r_{i}$ and the distance $d_{i}$ between the cell center $\left(x_{i}, y_{i}\right)$ and the fitted line

$$
\begin{aligned}
\Delta_{i} & =d_{i}-r_{i}, \\
d_{i} & =\frac{\left|A y_{i}+x_{i}+C\right|}{\sqrt{A^{2}+1}} .
\end{aligned}
$$

In other words, $\Delta_{i}=0$ means that the fitted line is tangent to the circle with the radius $r_{i}$ and the center at $\left(x_{i}, y_{i}\right)$. Figure 2 shows an example of a fitted muon trajectory. 


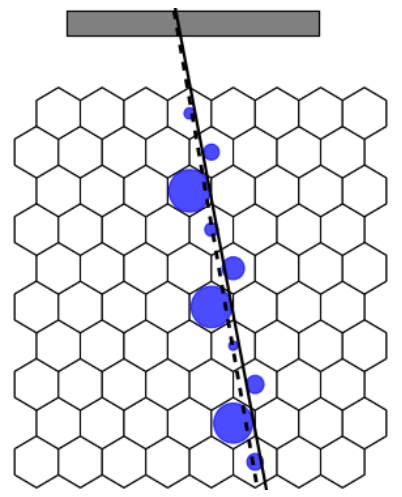

Fig. 2. A visualization of a reconstructed muon trajectory. The rectangle at the top of the picture represents the triggering scintillator. The circles originate in the centers of the cells which contributed to the event and their radii are calculated according to the $r_{i}(t)$ calibration function. The dashed line represents the prefit and the solid line represents the main fit.

\subsection{Iterative calibration procedure}

In order to increase the accuracy of the trajectory fitting, an iterative calibration was performed. During this procedure, muon trajectories are fitted for all events at every iteration step. The fit residua $\Delta$ are histogrammed by two indices: $i$ - the individual number of the cell giving the signal and $j$ - the number of the time subset $T_{j}$ containing the measured drift time $t_{i}$ (all subsets have the same length $\sim 14 \mathrm{~ns}$ ).

For each $\Delta_{i, j}$, a Gaussian function was fitted in order to find the distribution center. If this value is different from zero, the calibration function is shifted accordingly and the whole procedure is repeated. It was observed that five steps are sufficient for the calibration to converge. Figure 3 shows
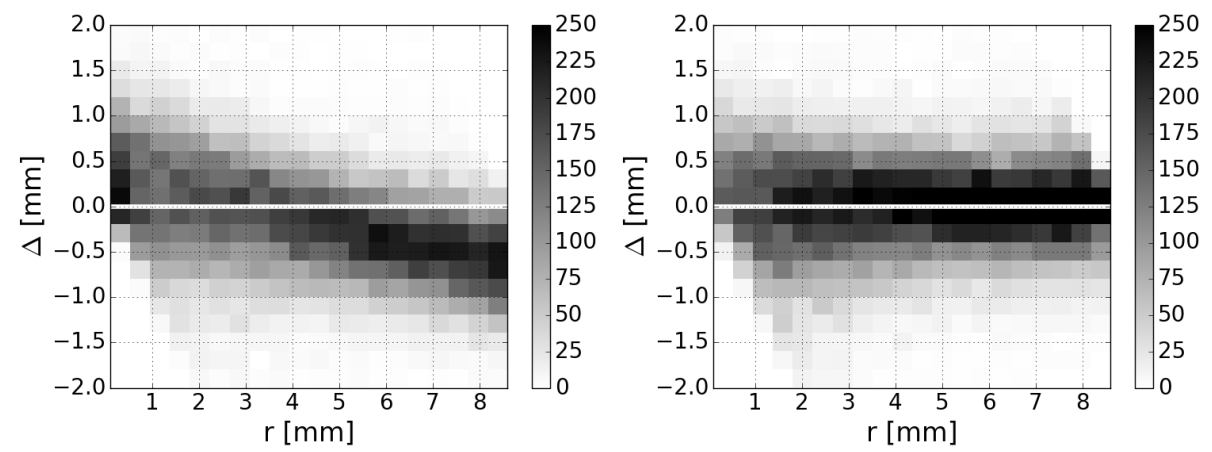

Fig. 3. An example of residua $\Delta_{i}$ distribution before (left) and after (right) the iterative calibration procedure. The systematic offset in the residua distribution is canceled after the calibration. 
the initial and the final residua distribution as a function of $r$. One can see that before the calibration procedure the largest systematic offsets are present at the cell periphery. The iterative calibration canceled these offsets in the whole range of $r$. Figure 1 (right) presents how the calibration function changed between the initial and the final iteration steps.

\section{Results}

\subsection{Resolution}

The single cell resolution $\sigma_{i, j}$ is defined as the standard deviation of the corresponding residua $\Delta_{i, j}$ distribution. Figure 4 (left) shows that these distributions are symmetric and can be reasonably approximated by a Gaussian function. Figure 4 (right) presents an example of the single cell resolution as a function of the distance from the anode wire, obtained from the measurement of cosmic muon trajectories. It decreases monotonically down to about $0.4 \mathrm{~mm}$ at $r=5 \mathrm{~mm}$ and stays constant at the cell periphery. This result agrees with that of Ref. [4] obtained for a planar cell operated at similar gas conditions. The resolution does not change after the iterative calibration since it only removes systematic offsets in the $r(t)$ function.
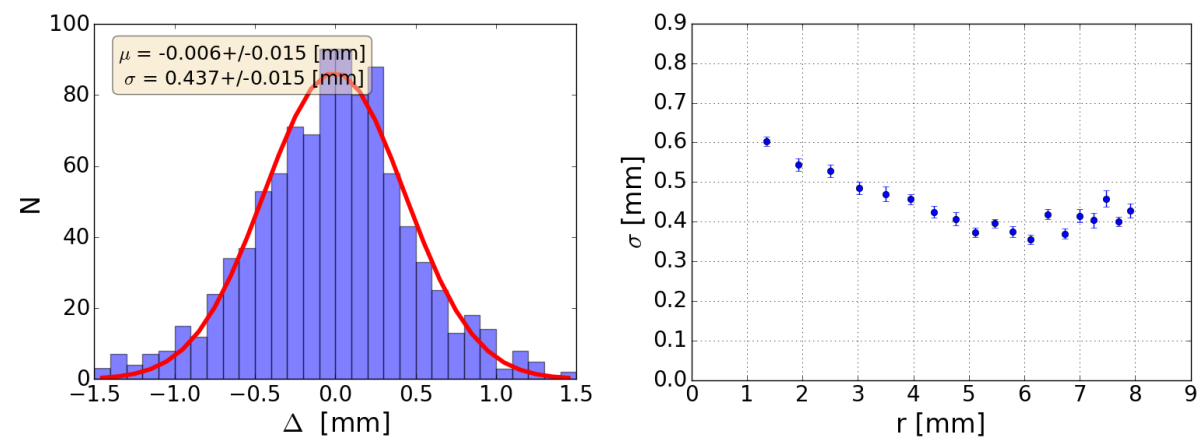

Fig. 4. Left panel: An example of residua $\Delta_{i, j}$ distribution from trajectory fits for a given drift times subset $T_{j}(t \in(314,327) \mathrm{ns})$ after the iterative calibration procedure. Right panel: Single cell resolution as a function of the distance from the cell center. The resolution is correlated with the velocity of the electron drift (slope of the $r(t)$ ) resulting in a higher position uncertainty in the region of stronger electric field (closer to the anode).

\subsection{Efficiency}

The location of the field wires (cathodes) in the hexagon corners makes the field distribution not fully symmetric. In particular, close to the field wires the electric field is weak and one expects there losses of primary ionization or its migration to neighboring cells. Moreover, additional field asym- 
metry can be observed for cells close to the chamber boundaries. In a first attempt, we studied the average cell efficiency as a function of the distance $r$ from the anode wire using cosmic muons penetrating the entire chamber.

For each fitted trajectory, a set of cells that the trajectory is crossing was selected. If the trajectory is crossing the cell $i$ at the distance $r$, the closest $r_{i, j}$ is found $\left(r_{i, j}=r_{i}\left(t_{j}\right)\right.$, where $t_{j}$ is the center of the $T_{j}$ subset) and a variable $N_{i, j, \text { total }}$ is incremented by one. If this cell delivered a signal

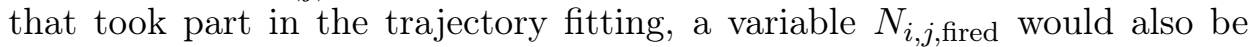
incremented by one. The single cell efficiency $\eta_{i}\left(r_{j}\right)$ is calculated as

$$
\eta_{i}\left(r_{j}\right)=\frac{N_{i, j, \text { fired }}}{N_{i, j, \text { total }}} .
$$

An example of the obtained efficiency function for one cell is presented in Fig. 5 (left).
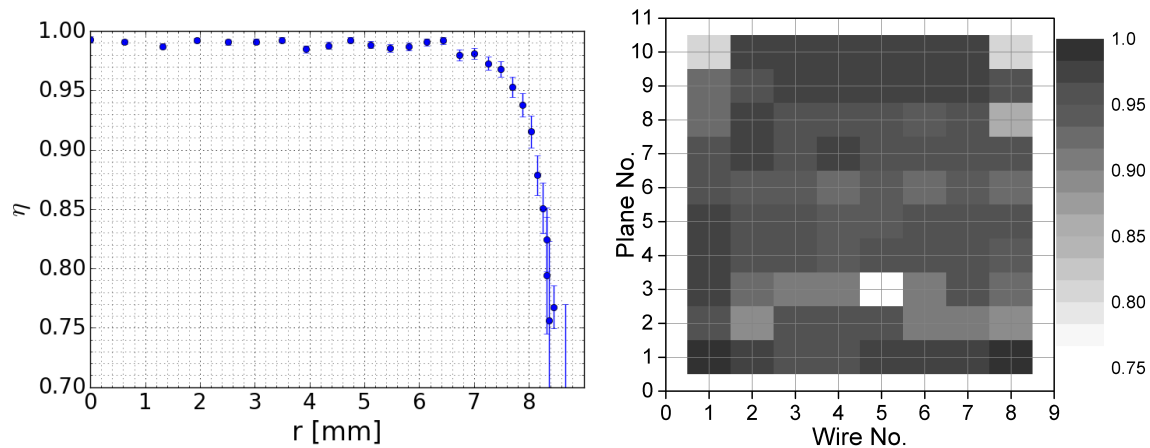

Fig. 5. Left panel: Single cell efficiency as a function of radius. Uncertainties at the edge of the cell are higher due to a lower statistics (bins have constant width in the $t$ dimension and not in $r$, which is getting smaller in a region of flattening of the $r(t)$ ); Right panel: Total cell efficiencies. The efficiency of the cell related to wire 5 in plane 3 is significantly lower than the rest due to a malfunction of a single readout channel, fixed afterwards.

A total cell efficiency $\bar{\eta}_{i}$ is defined as

$$
\bar{\eta}_{i}=\frac{\sum_{j} N_{i, j, \text { fired }}}{\sum_{j} N_{i, j, \text { total }}} .
$$

Total cell efficiency $\bar{\eta}_{i} \sim 0.95$ was reached for most of the cells (Fig. 5 (right)). Its uncertainty for most of the cells was $\sim 10^{-3}$. It is important to note that the relatively low total efficiency is caused by the cell periphery dominated by weak and nonsymmetric field. In almost the entire cell $(r<7 \mathrm{~mm})$ the efficiency is constant at the level of 0.98 or better. 


\section{Conclusions}

The preliminary results obtained from the run using cosmic muons as a source of the primary ionization in the miniBETA drift chamber are consistent with those obtained for the simplified setup [4]. A single cell spatial resolution in the drift plane of $\sigma_{i} \sim 0.4 \mathrm{~mm}$ and a total cell efficiency of $\bar{\eta}_{i} \sim 0.95$ (up to 0.98 for radii $r<7 \mathrm{~mm}$ ) were reached for most of the cells in the chamber filled with the mixture of helium-isobutane $(50 / 50)$ at 600 mbar. The gas mixture composition and its pressure will be further investigated to find the optimal conditions for the $\beta$-spectrum shape measurements. The trajectory finding algorithm was validated and it was shown that an iterative calibration procedure improves the fit performance. For a complete 3D trajectory fitting, the $Z$ coordinates of the trajectories (along the wires) have to be calculated according to the charge division technique. Its accuracy is under investigation. In the next step, implementing a pattern recognition algorithm is planed which will allow to distinguish the so-called V-tracks corresponding to backscattering events.

\section{REFERENCES}

[1] N. Severijns, J. Phys. G: Nucl. Part. Phys. 41, 114006 (2014).

[2] O. Naviliat-Cuncic, M. Gonzalez-Alonso, Ann. Phys. (Berlin) 525, 600 (2013).

[3] G. Soti, Ph.D. Dissertation, KU Leuven, 2013.

[4] K. Łojek et al., Nucl. Instrum. Methods Phys. Res. A 611, 284 (2009).

[5] K. Łojek et al., Nucl. Instrum. Methods Phys. Res. A 802, 38 (2015).

[6] G. Avolio et al., Nucl. Instrum. Methods Phys. Res. A 523, 309 (2004). 